\title{
The method to the madness of the cardiovascular consequences of alcohol
}

\author{
Ahluwalia $\mathrm{A}^{1}$, Jones $\mathrm{I}^{1}$, Haddad $\mathrm{N}^{1}$, Gwathmey $\mathrm{JK}^{2,3}$ and Haddad GE $^{1 *}$ \\ ${ }^{1}$ Howard University, College of Medicine, Department of Physiology \& Biophysics, USA \\ ${ }^{2}$ Boston University, School of Medicine, USA \\ ${ }^{3}$ Gwathmey Inc, MA, USA
}

\begin{abstract}
Alcohol consumption varies across cultures, populations, and generations. Excessive intake of alcohol has been linked to cardiovascular pathologies such as alcoholic cardiomyopathies (ACM). Understanding the mechanism by which alcohol associated cardiovascular disease occurs may allow the development of future targeted therapies. Additionally, exploration of the benefits of moderate alcohol consumption might help discern healthier guidelines for alcohol intake.

Background and aim: The purpose of this review is to specifically examine the cardiac and vascular effects of alcohol consumption. We will discuss the outcomes of low/moderate versus heavy alcohol drinking as well as the influence of drinking patterns, like daily, chronic intake, compared to quantity of alcohol consumption. The root of alcohol's negative consequences stems from its harmful direct and indirect effects on multiple organs at the cellular level. In this review, we will focus on the molecular etiology of alcohol-induced cardiac and vascular effects. With the widespread consumption of alcohol among individuals in the United States, along with the extreme health consequences that often follow alcoholism, it is important to determine in what ways alcohol use and abuse influences one's health as well as ways to safely and maybe beneficially consume alcohol. This review will particularly explore the specific effects of alcohol on cardiac and vascular function and examine the ways in which consumption can bestow beneficial effects. In understanding the mechanisms by which alcohol both harms and benefits organ systems, effective and targeted treatments can be developed.
\end{abstract}

\section{Introduction}

Alcohol is commonly consumed across countries and cultures but can have detrimental health effects if used in excess. Harmful and even fatal outcomes such as heart failure, stroke, and alcoholic cardiomyopathy may result from alcohol abuse. However, various studies show that if certain forms of alcohol are consumed consistently in moderation, positive health effects may result [1]. Everything we can consume could carry detrimental as well as beneficial effects depending on threshold and tolerance levels, and alcohol is no exception. Thus, it is imperative to understand the consequences that alcohol can have on one's health. Furthermore, because alcoholism is the fourth leading preventable cause of death in the U.S. and fifth leading risk factor for premature death worldwide, with an approximate 88,000 and 3.3 million annual deaths respectively [2], it is important to determine whether there can be guidelines established for alcohol intake in an effort to derive beneficial effects from alcohol consumption.

In a large-scale study aimed at addressing the lack of information on alcohol use and abuse patterns, the National Institute on Alcohol Abuse and Alcoholism conducted the National Epidemiologic Survey on Alcohol and Related Conditions (NESARC) comprised of a sample population that included people of varying ages and races. With a sample size of 43,093 people and an 81 percent response rate, the NESARC can be used to gauge the prevalence of alcohol consumption across specific groups in the United States. Before analyzing the data produced, however, it is important to define alcohol abuse and alcohol dependence. Specific criteria include clinically significant use and distress as a result of alcohol consumption within a 12-month timeframe in alcohol abuse as compared to alcohol dependence which is marked by withdrawal symptoms in addition to an increased tolerance in the same timeframe [3]. Both alcohol abuse and alcohol dependence have been combined under the category of alcohol use disorder (AUD) in the DSMV. Symptoms of AUD include physical dependence upon, increased tolerance for, and a craving for alcohol [3].

As a whole, NESARC [4] noted that alcohol abuse has increased by 1.63 percent while alcohol dependence has been reported to have decreased by 0.57 percent between 1991-1992 and 2001- 2002. Both men and women have experienced an increased in abuse, with the most notable increases of abuse occurring in young Black and Hispanic individuals. On the other hand, alcohol dependence has been reported to have increased among men, young black women, and Asian men in this time period. In addition to highlighting the prevalence of alcohol abuse and dependence across various ethnic, age and gender groups, analysis of the NESARC demonstrates why these findings are significant to one's health.

It has been shown that people who begin drinking at a young age are more likely to abuse or gain health problems resulting from alcohol later in life. Indeed, researchers determined that 45 percent of individuals who began drinking before age 14 developed alcohol dependence later in life, whereas 10 percent of individuals who only began drinking after age 21 became alcohol dependent [4]. With the rise

Correspondence to: Haddad GE, Department of Physiology \& Biophysics College of Medicine, Howard University, NW, Washington DC 20059, USA; E-mail: ghaddad@howard.edu

Received: January 26, 2018; Accepted: February 02, 2018; Published: February 09,2018 
in alcohol abuse and dependence noted particularly among younger individuals, intervention is of significant importance especially in light of health concerns it can cause later in life.

Chronic alcohol abuse specifically can elicit a plethora of effects, such as, myocyte hypertrophy, fibrosis, mitochondrial degeneration, fibrosis, modification of contractile proteins, and disruption of calcium homeostasis [5]. Various cardiomyopathies such as ventricular dilation, hypertrophy, and even heart failure have been associated with chronic alcohol consumption [6]. These all fall under the category of alcoholic cardiomyopathies (ACM) [7]. One way in which alcohol has been shown to dysregulate cardiac function is its effect on protein metabolism. Interestingly, chronic alcohol usage results in impaired mRNA initiation and elongation during translation, whereas acute alcohol intake seems to inhibit initiation during translation alone [8-11]. However, both result in abnormal protein metabolism that eventually leads to impaired contractility at the level of the cardiomyocytes. On the vasculature side, alcohol is also associated with an increase in total cholesterol, increased LDL, cholesterol, triglycerides, and an increase in the 10-year coronary artery disease (CAD) risk [12].

\section{Discussion of the Cardiovascular Consequences of Alcohol}

Alcohol consumption is a very common pastime in Western countries, with $53 \%$ of Americans regularly consuming alcohol and $44 \%$ of drinkers consuming greater than 5 standard drinks on a single occasion [13]. Binge drinking is prevalent in those that regularly consume alcohol. Unfortunately, excessive alcohol consumption and binge drinking can have detrimental effects on cellular function via a plethora of mechanisms. Due to its intricate and elaborate electrophysiological pathways, the cardiovascular system is particularly susceptible to alcohol- related damage. Owing to alcohol's epidemiology and harmful effects, it is imperative to explore and characterize what severe drinking is, and the ways in which it affects the cardiovascular system. However, it is also important to note that low-to-moderate level consumption of alcohol has been shown to reduce mortality in other pathological conditions, which will further be discussed as well. Due to the clear differences in the effects that alcohol can inflict based upon dose and manner of consumption, it is important to distinguish between the physiological and molecular changes that can arise with different types of drinking.

\section{Severe drinking}

Two subsets lie under the category of severe drinking: chronic alcoholism and binge alcohol consumption. These two categories express drinking in the terms of the frequency of drinking (daily, chronic drinking) and drink volume (binge drinking). Binge drinking can be characterized as the consumption of 4 to 8 servings of alcohol on a single occasion [14]. In contrast, chronic consumption of alcohol is characterized in terms of frequency i.e. most often daily intake [14]. Studies have shown that chronic alcohol consumption and binge drinking can subject the heart to non-ischemic dilated cardiomyopathy, electrical and conduction disturbances, and may lead to sudden cardiac death [15].

Exploration of the different effects of dose-related alcohol consumption on the heart is necessary, as alcohol consumption may be an important risk factor for atrial fibrillation as obesity [13]. In a cohort of 8,602 subjects, the HR of developing AF was 2.68 for heavy drinkers ( $\geq 40$ standard drinks/week), compared with 1.72 for obesity and 1.02 for hypertension [13]. Alcohol may also play a more significant role in other forms of cardiomyopathy than is currently understood. As most chronic alcohol-consumers are also occasionally binge drinkers, a synergistic pathophysiological effect may result. This additive effect should be explored in hopes of obtaining a better understanding and identification for pure alcohol-induced cardiomyopathy.

\section{High volume alcohol consumption}

Acute consumption of a large volume of alcohol, binge drinking, is an activity in which many cultures across the world participate. Binge drinking is a pastime that often represents celebration, and typically occurs around the holidays or during college students' social activities. Those that partake in binge drinking are frequently young and without evidence of heart disease. In 1978, Ettinger coined the term "Holiday Heart Syndrome" (HHS) that describes this phenomenon. HHS is defined as temporarily abnormal heart rhythms that occur in relatively healthy patients after excessive alcohol consumption [16]. Those that suffer from HHS report palpitations, chest pain, and dyspnea on weekends or after holidays-hence the term "holiday heart syndrome" [5]. Since the characterization of this term in 1978, volume-relatedalcohol-induced pathologies in acute settings have continued to be explored in hopes of understanding the interaction between binge drinking, chronic alcohol consumption, and cardiomyopathies.

Greater volumes of alcohol consumption are positively associated with increased left ventricular volume and total left ventricular mass and have an inverse relationship with global left ventricular ejection fraction [15]. In a study published by Hung et al 2018, subjects who consumed excessive amounts of alcohol presented with markedly enlarged left ventricles, a greater left ventricular mass index, and reduced left ventricular ejection fraction compared to the light-tomoderate drinker populations [15]. Additionally, excessive volumes of alcohol in an acute setting have been shown to demonstrate increased serological markers of myocardial injury and cardiovascular inflammation. Changes in biochemical and ultrastructural indices of myocardial dysfunction, metabolism, blood pressure, heart rate, coagulation processes, and coronary vasoconstriction are all seen with heavy, acute drinking [17]. These changes can be partially explained by the observation that binge drinking is known to induce myocardial inflammation and to increase sympathetic tone. To that effect, in healthy non-alcoholics, there was a $17 \%$ increase in heart rate 12 hours post-binge [13]. In addition, an increase in serum troponin levels and atrial tachyarrhythmias have been reported [13]. In rabbits, acute heavy alcohol consumption initiates electrical atrial remodeling. A 5-day alcohol infusion reduced L-type calcium channels and sodium current density, the acetylcholine- sensitive potassium channel Kir3.1 was upregulated in rat atria and there was an increase in the alcohol metabolite, acetaldehyde [13]. The increase in the acetylcholinesensitive potassium channel activity promotes repolarization and shortens action potential duration [13]. Furthermore, in rabbit pulmonary vein myocytes, alcohol administration also shortened the pulmonary vein action potential duration by increasing Ito outward potassium current activity. Being that a common cause of atrial ectopic beats has been shown to be related to pulmonary vein dysfunction $[18,19]$ this is a significant finding in understanding the mechanism of arrhythmias in binge alcohol consumption and might be particularly important in pathogenesis of atrial fibrillation $[20,21]$.

According to a study published in the Journal of the American College of Cardiology, binge drinking combined with habitual moderate consumption was associated with a similar atrial fibrillation 
risk as chronic heavy drinking [13]. Although binge drinking is more common than chronic alcohol dependence [2], the amount of attention paid to its effects does not equate to its commonality. The continuance of research on the effects of binge drinking is particularly important due to its cultural and age-related frequency.

\section{Chronic drinking}

Chronic alcohol abuse has significant effects on the cardiovascular system as acute binge drinking. Subjects who had habitual, daily alcohol intake ( $\geq 90 \mathrm{~g} /$ day) were found to have a higher resting heart rate, wider QRS duration, longer QT interval, and a trend towards greater proportion of left ventricular hypertrophy [15]. Habitual alcohol intake, independent of dosage, showed a graded increase of fragmented QRS (fQRS) percentage compared to the nondrinkers [15]. As widened or fQRS has been discovered to be a potential phenotypic marker of cardiovascular mortality [22], this finding is particularly noteworthy.

A similar study emulating habitual alcohol intake was conducted in mice over the course of 14 weeks. There was a control group, a group given $4 \%$ alcohol, and a group given $6 \%$ alcohol. Compared to the control group, the alcohol-consuming mice had a lower body weight but higher heart-weight-to-body-weight ratio. They had significantly larger left ventricular chamber size and increased left ventricular mass. Ventricular cardiac myocytes had significant hypertrophy, which is indicative of a precipitating cardiomyopathy [15].

Cellular transformations occurred in the mice as well, specifically in proteins that are essential for the electrophysiological homeostasis of the heart. Gap junction protein connexin $43(\mathrm{Cx} 43)$ and sodium channel subunit Nav1.3 are important proteins in determining the propagation of cardiac myocyte electrical impulses [23]. In a healthy heart, gap junctions assembled from connexin proteins (connexin 43 being the most predominant) allow for the movement of currents between the cardiac myocytes (i.e. electrical coupling) [23]. Remodeling and down-regulation of $\mathrm{Cx} 43$ commonly occurs with cardiomyopathies and arrhythmias [23]. Changes in $\mathrm{Cx} 43$ and Nav1.5 proteins were observed in the alcohol-consuming mice (both the $4 \%$ and $6 \%$ alcohol groups), as compared to the control. Western blotting and immunoconfocal microscopy demonstrated greater than a $30 \%$ reduction in the sodium channel for the $4 \%$ alcohol group, and a $50 \%$ decrement in the $6 \%$ alcohol group compared to control mice. Furthermore, in order to observe the changes in distribution and location of the sodium-ion channels, the cytoplasm and cell membranes were examined in the alcohol-fed and control mice. The alcohol-fed mice had 40-50\% more $\mathrm{NaV} 1.5$ proteins in the cytoplasm than the control, demonstrating diminished membrane NaV1.5 distribution [15]. Optical mapping showed that the alcohol groups had a slower propagation of action potential than the control by $43-59 \mathrm{~cm} / \mathrm{s}$. The $6 \%$ alcohol group's action potentials were characterized by re-entry or less uniform activation directions, which creates a milieu for cardiomyocyte arrhythmias [15].

Gap junction remodeling in mice consuming alcohol chronically (indicated by downregulation of $\mathrm{Cx} 43$ proteins) combined with an increase in the total expression of $\mathrm{NaV} 1.5$ protein (which aggregated in the cytoplasm and showed decreased membrane expression) may play significant role in electrophysiological pathologies seen with chronic/ heavy alcohol usage. These observations suggest that heavy and or/ long-term alcohol consumption can damage primary ion channels involved in the propagation of action potentials in cardiomyocytes. Additionally, it can also dysregulate the function of pulmonary vascular smooth muscle cells, as alterations in these structural components of electrical properties contribute to electrical and mechanical asynergy and thereby inefficient contractile function [24]. Abnormalities in action potentials can lead to homeoadaptive responses that further elicit arrhythmias and re-entry formations. The mouse model of chronic alcohol consumption provides insights into changes at the cellular level that underly electrophysological changes in cardiac myocytes as well as vascular smooth muscle cells.

Long-term alcohol abuse is not only linked to reduction in sodium channel density but also to a decrease in intracellular calcium concentration and adenosine triphosphate. Specifically, intracellular calcium plays a pivotal role in the regulation of cardiomyocyte contraction. Likewise, acetaldehyde and ethyl esters, alcohol's primary metabolites, have the ability to uncouple excitation-contraction coupling. This promotes oxidative damage and lipid peroxidation, decreasing the expression of myofibrillar proteins and isoforms of myosin. In conjunction with the homeostatic mechanisms of compensation (renin-angiotensin-aldosterone system, ANP, and sympathetic nervous system), alcohol-induced cardiomyopathy manifests in the forms of left ventricular dilation, hypertrophy of cardiomyocytes, and electrophysical myopathies [5]. Cellular and sympathetic nervous system changes can act synergistically in mediating electrophysiological effects on the heart [13].

In patients with atrial fibrillation (AF), coagulopathies have been identified as an alcohol- mediated sequela in those who drank heavily. Male heavy drinkers with AF (>27 standard drinks/week) were found to be at a significantly higher risk for thromboembolism [13]. The definition of "heavy" alcohol consumption here is distinct-a combination of excessive alcohol intake with recurring daily frequency. It is often difficult to separate the intake volume from frequency because of unreliable reporting and societal norms as previously mentioned.

The relationship between AF patients, dose-related alcohol consumption, and coagulopathies remains poorly understood. However, a Korean study assessed the significance of alcohol consumption as a risk factor for coronary artery spasm. The alcoholconsuming group in a pool of patients with angina had a significantly higher incidence of coronary artery stenosis, spontaneous spasm, and myocardial bridging (when a coronary artery runs through the myocardium instead of lying on top of it) compared to the nondrinking group [25]. They had severe minimal narrowing diameter and a higher incidence of multivessel spasm (including the left circumflex coronary artery, a major contributor of blood supply to the bundle of His branches and the intracardiac conducting system) compared to the non-drinking group [25]. Those that were defined as excessive drinkers had an ever-higher risk for coronary artery spasm (CAS) [25].

\section{Moderate drinking}

In contrast to the serious complications that may arise from the different forms of severe drinking, various health benefits or reductions in disease progression have been demonstrated in studies on moderate drinking. Ethanol itself has been shown to exert protective effects on the cardiovascular system $[26,27]$. The most widely known component of an alcoholic beverage that can confer some additive protection is resveratrol, which is found in wine. As an antioxidant, resveratrol is able to relieve some of the oxidative stress placed on cardiomyocytes and thus can halt the progression of cardiac disease such as alcoholic cardiomyopathies [28].

Overall, moderate drinking can be thought to be split into two categories: a small amount of alcohol consumed daily, or a small amount of alcohol consumed episodically. This latter definition would 
need to be distinguished from binge drinking, as defined in a previous section of this review. However, since some difficulty lies in collecting information about episodic "small" amounts of drinking, many studies on low-level alcohol consumption choose to define moderate drinking in line with the first category stated above. Klatsky et al. [29] for instance, studied the effects of alcohol on cardiovascular disease (CVD) in individuals who consumed three or fewer drinks per day, with about $0.5 \mathrm{oz}$ of alcohol per drink. Mortality curves were plotted based on subjects that practiced abstinence (including both lifelong abstainers or former drinkers) as well as subjects that continued drinking various amounts of alcohol. A U-shaped mortality curve resulted, where the lowest risk group for CVD deaths was a set of subjects that drank 1-2 drinks per day, and the lowest risk group for specifically coronary artery disease (CAD) associated deaths were individuals that drank 3-5 drinks per day.

On the other side of the coin, one study showed that discontinuation of alcohol consumption in a specific population worsened their disease outcomes. Uribe et al. performed a metabolic assessment of a group of non-diabetic and non-cirrhotic Latino subjects before and after either allowing one group to consume moderate amounts of alcohol or discontinuing alcohol use altogether in another group. Although each patient's baseline alanine aminotransferase (ALT) levels, a marker of liver function, seemed to play a role in the outcome, an increase in hepatic insulin resistance was seen to increase in subjects that discontinued use of alcohol versus those that continued to drink alcohol in moderate quantities. Age was another variable that seemed to be at play; the younger patients in the study reaped greater metabolic benefits from their low-level alcohol consumption than the older subjects, indicating another factor that may influence how positively one may respond to moderate alcohol use. Overall, the curve between alcohol consumption and insulin resistance was also found to be U-shaped, with both abstinence and severe alcohol use worsening health outcomes for these patients more than moderate use. Although this study does not directly discuss the cardiovascular benefits that alcohol may or may not provide, it does present a systemic disease process that progressed more rapidly when alcohol use was discontinued. It may also serve as a model for potential future studies on CVD and moderate alcohol consumption by focusing on the other perspective of the effects of discontinuation, rather than continued consumption, of alcohol.

Since studies on the effects of moderate drinking vary greatly and often use unique variables, more standardized research is needed to define whether a study truly shows beneficial effects that result from low amounts of alcohol consumption. A study by Helander et. al demonstrates this importance of standardization of variables in alcohol research [30]. However, despite there being a lack of information to concisely define the parameters under which moderate alcohol consumption can be beneficial, the studies discussed above show compelling evidence that moderate intake may indeed influence positive health outcomes. With the idea that consuming alcohol in moderation can be beneficial to overall health and disease outcomes in mind, we can explore the effects of alcohol on the heart and the vasculature at a more molecular level. The main ways that ethanol consumption has been demonstrated to improve cardiovascular health is through its effects on cholesterol levels, platelet aggregation, and vasodilation [31-37].

An age-adjusted study observed the effects that alcohol consumption had on blood pressure, HDL, and LDL levels in a cohort of 1,768 Japanese men under the Honolulu Heart Program [34]. Alcohol use was found to lower LDL and cholesterol levels as well as increase HDL. With atherosclerosis being a frontrunning player in perpetuating cardiovascular damage and its sequelae, this decrease in "bad" cholesterol and increase in "good" cholesterol should not be overlooked. However, its concurrent effect on increasing blood pressure, which is a negative contributor to cardiovascular health, cannot be ignored either. Nevertheless, these researchers noted an overall U-shaped mortality curve with low alcohol usage, just as in previous studies we have discussed. The benefit that lower LDL levels provided was found to essentially be cancelled out by the increased mortality associated with higher systolic blood pressure in the statistical model used to analyze the data. Therefore, they calculated about half of the improvement resulting in the U-shaped curve was likely to be due to increased HDL2 and HDL3 levels. The other half of the benefit incurred by low alcohol use was unexplained, but suspected to be due to alcohol's hemostatic effects, some of which will be discussed in an upcoming section. It was also noted that although this study was performed on a specific population, other studies achieved similar results in varying cohorts, providing support that the observed benefits were not cohort-specific and the results would be replicated in another population $[32,34]$.

A number of other studies have accounted for the effects of moderate alcohol intake on various proteins and blood-related factors. Tissue-type plasminogen activator (t-PA) is one such factor. As a serine protease that is responsible for lysing fibrin, a major component of blood clots, greater t-PA levels can counter the physiological effects of thrombosis and emboli formation. Ridker et al. found that increased plasma concentrations of t-PA were associated with moderate alcohol consumption even after adjusting for other variables such as the increased HDL levels that result from moderate alcohol intake [36].

An interesting study performed by Hendriks et al. demonstrated that tissue plasminogen activator ( $t$-PA) and plasminogen activator inhibitor (PAI), which counters the effects of $t-P A$, follow a circadian rhythm. This study took real-life situations into consideration by measuring fibrinolytic processes after alcohol was consumed with a meal. It is important to emulate this because most moderate drinkers indeed consume alcohol with meals. He found that t-PA bounced back to a significantly higher level the next morning after dinner with moderate alcohol consumption. This is important because many studies exploring CAD and t-PA do not take the effects of daily occurrences, like drinking alcohol with a meal or the normal circadian rhythm of fibrinolytic factors, into consideration. These effects observed in the early morning are consistent with a decrease in risk of CAD in moderate drinkers [31,33].

Besides these effects on the fibrinolytic pathway, other research on alcohol's hemostatic effects found that at low doses, ethanol caused a decrease in thromboxane A1 and increase in prostacyclin levels in human blood [35]. As a potent inhibitor of platelet aggregation [38], increased prostacyclin offers another protective factor against CVD in a similar fashion that increased levels of t-PA does. Even more interestingly, use of $400 \mathrm{mg} \%$ ethanol did not show a consistent pattern in inhibiting platelet aggregation, but much lower concentrations demonstrated potentiation of prostacyclin activity [35].

Additionally, alcohol generally causes vasodilation of both arteries and veins in vivo and in vitro [39]. One study found that moderate intake of alcohol increased the diameter of the brachial artery at both resting conditions and during reactive hyperemia [37]. This has important implications since it indicates low levels of alcohol consumption can improve blood flow after ischemic episode(s) related 
to a cardiovascular pathology. Although the study aimed to determine whether endothelial function was the direct cause of such vasodilation, it was suggested that alcohol's stimulation of the central nervous system may have played a role in the vasodilation observed. However, the production of prostacyclin by endothelial cells and its vasodilatory effects provides reasonable hypothesis of prostacyclin playing a role in alcohol-induced vasodilation [37], though findings were inconclusive. If so, this could have played a role in previous finding [35] that increased prostacyclin in response to alcohol led to vasodilatory effects, as discussed above. Once again, these findings support the hypothesis that despite the havoc alcohol abuse can wreak on one's health, it has the potential to benefit the cardiovascular system if consumed in moderate quantities.

\section{Limitations and future directions}

The prevalence and identification of alcohol induced cardiomyopathy is often difficult to clinically identify and to characterize. Mirijello et al. elaborates the gaps and different variables that challenge the ability to perform conclusive research as well as aims to discern potential benefits of ethanol. Firstly, the extent of alcoholic cardiomyopathy is determinant on the amount of daily alcohol ingested, and the duration of alcohol abuse [23]. The amounts are most often patient-reported thereby making the dosages required for the development and manifestation of ACM difficult to determine. Although most studies adjust for confounding variables, it is difficult to isolate the extent of alcohol-induced cardiomyopathy in the presence of conditions like diabetes, hypertension, and autoimmune disease. Additionally, alcohol's effects on each individual are multivariate. Juxtaposition of genetic and non-genetic factors play a pivotal role on the pathogenesis of alcoholic cardiomyopathy. For example, alcohol dehydrogenase alleles, HLA-B8, thiamine deficiency, and exposure to different cardiotoxic substances are examples that influence the impact of alcohol consumption on cardiovascular outcomes [5]. These implications are important to consider when designing clinical trials or evaluating clinical outcomes. Nevertheless, despite differing factors, clinical challenges, and uncertainty about the actual amount of ethanol that mediates alcohol-induced toxicity to cardiomyocytes as well as vascular muscle cells, there appears to be sufficient concrete data that indicates that alcohol can be cardiotoxic in high quantities and pose certain benefits in low quantities.

Alcohol consumption is historically popular amongst a wide array of age groups, cultures, and backgrounds. Research in this area would be invaluable as it would offer individuals a healthy avenue to consume alcohol without insisting on complete abstinence. Nevertheless, despite having conducted a number of studies that have explored beneficial effects of alcohol consumed in moderation, clear parameters on what dose best balances alcohol's cardioprotective effects over its negative consequences have yet to be established. Additionally, a clear demonstration of the cardiovascular as well as larger systemic effects that moderate intake of alcohol can impart requires further study.

Greater medical compliance might be attained with drinking in moderation rather than acute abstinence which often results in withdrawal symptoms in patients and leads to a high level of noncompliance resulting in continued alcohol abuse.

\section{Acknowledgement}

This work was supported in part by grants 1 R15 AA019816-01A1 and 2G12 RR003048 RCMI, Division of Research Infrastructure, BFSAP Howard University College of Medicine to GEH. Anchal Ahluwalia and India Jones have equal contribution to this work.

\section{Conflict of Interest}

The authors declare that there is no conflict of interest regarding the publication of this paper.

\section{References}

1. Rehm J, Roerecke M (2017) Cardiovascular effects of alcohol consumption. Trends in Cardiovascular Medicine 27: 534-538.

2. Alcohol Facts and Statistics (2017) National Institute on Alcohol Abuse and Alcoholism.

3. Alcohol Use Disorder: A Comparison Between DSM-IV and DSM-5 (2016) National Institute on Alcohol Abuse and Alcoholism.

4. National Epidemiologic Survey on Alcohol and Related Conditions. National Institute on Alcohol Abuse and Alcoholism.

5. Mirijello A, Tarli C, Vassallo GA, Sestito L, Antonelli M, et al. (2017) Alcoholic cardiomyopathy: What is known and what is not known. Eur J Intern Med 43: 1-5. [Crossref]

6. Krenz M, Korthuis RJ (2012) Moderate ethanol ingestion and cardiovascular protection from epidemiologic associations to cellular mechanisms. J Mol Cell Cardiol 52: 93104. [Crossref]

7. Steiner JL, Lang CH (2017) Alcoholic cardiomyopathy: Disrupted protein balance and impaired cardiomyocyte contractility. Alcohol Clin Exp Res 41: 1392-1401. [Crossref]

8. Lang CH, Frost RA, Kumar V, Vary TC (2000a) Impaired myocardial protein synthesis induced by acute alcohol intoxication is associated with changes in eIF4F. Am J Physiol Endocrinol Metab 279: E1029-1038. [Crossref]

9. Lang CH, Frost RA, Kumar V, Wu D, Vary TC (2000b) Impaired protein synthesis induced by acute alcohol intoxication is associated with changes in eIF4E in muscle and eIF2B in liver. Alcohol Clin Exp Res 24: 322-331. [Crossref]

10. Lang CH, Wu D, Frost RA, Jefferson LS, Kimball SR, et al. (1999) Inhibition of muscle protein synthesis by alcohol is associated with modulation of eIF2B and eIF4E. Am J Physiol 277: E268-276. [Crossref]

11. Vary TC, Jefferson LS, Kimball SR (2001a) Insulin fails to stimulate muscle protein synthesis in sepsis despite unimpaired signaling to 4E-BP1 and S6K1. Am J Physiol Endocrinol Metab 281: E1045- 1053. [Crossref]

12. Foerster M, Marques-Vidal P, Gmel G, Daeppen J, Cornuz J, et al. (2009) Alcohol Drinking and Cardiovascular Risk in a Population with High Mean Alcohol Consumption. Am J Cardiol 103: 361-368. [Crossref]

13. Voskoboinik A, Prabhu S, Ling L, Kalman JM, Kistler PM (2016) Alcohol and Atrial Fibrillation. Journal of the American College of Cardiology 68: 2567-2576.

14. Biagioni N, Pettigrew S, Jones S, Stafford J, Daube M, et al. (2017) Defining binge drinking: young drinkers perceptions of risky alcohol consumption. Public Health 152 55-57. [Crossref]

15. Hung C, Lai Y, Chi P, Chen L, Tseng Y, et al. (2018) Dose-related ethanol intake, Cx43 and Nav1.5 remodeling: Exploring insights of altered ventricular conduction and QRS fragmentation in excessive alcohol users. J Mol Cell Cardiol 114: 150-160. [Crossref]

16. Ettinger PO, Wu CF, Cruz CD, Weisse AB, Ahmed SS, et al. (1978) Arrhythmias and the "Holiday Heart": Alcoholassociated cardiac rhythm disorders. Am Heart J 95: 555-562. [Crossref]

17. Waszkiewicz N, Szulc A, Zwierz K (2013) Binge Drinking-Induced Subtle Myocardial Injury. Alcohol Clin Exp Res 37: 1261-1263. [Crossref]

18. Chen Y, Chen S, Chen Y, Tai C, Chan P, et al. (2004) Effect of ethanol on the electrophysiological characteristics of pulmonary vein cardiomyocytes. Eur $J$ Pharmacol 483: 215-222. [Crossref]

19. Haïssaguerre M, Jaïs P, Shah DC, Takahashi A, Hocini M, et al. (1998) Spontaneous Initiation of Atrial Fibrillation by Ectopic Beats Originating in the Pulmonary Veins. $N$ Engl J Med 339: 659-666. [Crossref]

20. Hart RG, Pearce LA, Mcbride R, Rothbart RM, Asinger RW (1999) Factors Associated With Ischemic Stroke During Aspirin Therapy in Atrial Fibrillation : Analysis of 2012 Participants in the SPAF I III Clinical Trials. Stroke 30: 1223-1229. [Crossref]

21. Planas F, Romero-Menor C, Vázquez-Oliva G, Poblet T, Navarro-López F (2006) Natural History of and Risk Factors for Idiopathic Atrial Fibrillation Recurrence (FAP Registry). Rev Esp Cardiol 59: 1106-1112. [Crossref] 
22. Desai AD, Yamazaki T, Kaykha A, Chun S, Froelicher V (2006) Prognostic significance of quantitative QRS duration. Am J Med 119: 600-606. [Crossref]

23. Severs NJ, Bruce AF, Dupont E, Rothery S (2008) Remodelling of gap junctions and connexin expression in diseased myocardium. Cardiovasc Res 80: 9-19. [Crossref]

24. Spragg DD (2003) Regional Alterations in Protein Expression in the Dyssynchronous Failing Heart. Circulation 108: 929-932. [Crossref]

25. Sohn SM, Choi BG, Choi SY, Byun JK, Mashaly A, et al. (2018). Impact of alcohol drinking on acetylcholine-induced coronary artery spasm in Korean populations. Atherosclerosis 268: 163-169. [Crossref]

26. Ippolito JA, Curtis BJ, Choudhry MA, Kovacs EJ, Haddad GE (2013) Alcohol and immunology: Summary of the 2012 Alcohol and Immunology Research Interest Group (AIRIG) meeting. Alcohol 47: 589-593. [Crossref]

27. Uribe LA, Bacchetti P, Gelman N, Burchard E, Fitch M, et al. (2017) Impact of Moderate Alcohol Discontinuation on Insulin Action and Secretion in Latinos With and Without Hepatitis C. Alcohol Clin Exp Res. [Crossref]

28. Spanier G, Xu H, Xia N, Tobias S, Deng S (2009) Resveratrol reduces endothelial oxidative stress by modulating the gene expression of superoxide dismutase 1 (SOD1), glutathione peroxidase 1 (GPX1) and NADPH oxidase subunit (NOX4). $J$ Physiol Pharmacol 60: 111-116. [Crossref]

29. Klatsky AL (1999) Moderate drinking and reduced risk of heart disease. Alcohol Res Health 23: 15-23. [Crossref]

30. Helander A, Wielders J, Anton R, Arndt T, Bianchi V, et al. (2017) Reprint of Standardisation and use of the alcohol biomarker carbohydrate-deficient transferrin (CDT). Clin Chim Acta 467: 15-20. [Crossref]
31. Arranz S, Chiva-Blanch G, Valderas-Martínez P, Medina-Remón A, LamuelaRaventós, et al. (2012) Wine, Beer, Alcohol and Polyphenols on Cardiovascular Disease and Cancer. Nutrients 4: 759-781. [Crossref]

32. Criqui MH, Cowan LD, Tyroler HA, Bangdiwala S, Heiss G, et al. (1987) Lipoproteins as mediators for the effects of alcohol consumption and cigarette smoking on cardiovascular mortality: Results from the Lipid Research Clinics Follow-up Study Am J Epidermiol 126: 629-637. [Crossref]

33. Hendriks HF, Veenstra J, Wierik EJ, Shaafsma G, Kluft C (1994) Effect of moderate dose of alcohol with evening meal on fibrinolytic factors. Bmj 308: 1003-1006. [Crossref]

34. Langer RD, Criqui MH, Reed DM (1992) Lipoproteins and blood pressure as biological pathways for effect of moderate alcohol consumption on coronary heart disease. Circulation 85: 910-915. [Crossref]

35. Mehta P, Mehta J, Lawson D, Patel S (1987) Ethanol stimulates prostacyclin biosynthesis by human neutrophils and potentiates anti-platelet aggregatory effects of prostacyclin. Thromb Res 48: 653-661. [Crossref]

36. Ridker PM (1994) Association of moderate alcohol consumption and plasma concentration of endogenous tissue-type plasminogen activator. JAMA 272: 929-933. [Crossref]

37. Vlachopoulos C, Tsekoura D, Tsiamis E, Panagiotakos D, Stefanadis C (2003) Effect of alcohol on endothelial function in healthy subjects. Vasc Med 8: 263-265. [Crossref]

38. Radomski M, Palmer R, Moncada S (1987) The anti-aggregating properties of vascular endothelium: interactions between prostacyclin and nitric oxide. Br J Pharmacol 92 639-646. [Crossref]

39. Altura BM, Altura BT (1983) Peripheral vascular actions of alcohol and its interaction with neurohumoral substances. Neurobehavioural Toxicology and Teratology 5: 211-220.

Copyright: (C2018 Ahluwalia A. This is an open-access article distributed under the terms of the Creative Commons Attribution License, which permits unrestricted use, distribution, and reproduction in any medium, provided the original author and source are credited. 